\title{
I'm Virtually a Psychiatrist: Problems with Telepsychiatry in Training
}

\author{
J. Alexander $S \operatorname{cott}^{1}$ (1)
}

Received: 12 August 2021 / Accepted: 31 August 2021 / Published online: 9 September 2021

(c) Academic Psychiatry 2021

Admittedly, I've never liked telemedicine. When I first observed it in medical school, I found it impersonal and artificial. But with the COVID-19 pandemic, what was once an alternative became the only game in town. Although optimists have noted the virtues of virtual visits (e.g., improved access to care, our better understanding of patients' environments), practicing nearly exclusively in this way for a year has frustrated me. In telepsychiatry, that interaction between patient and psychiatrist which is so fundamental to the therapeutic process is altered in many ways [1]. I worry that these alterations have a negative impact on my learning.

Telepsychiatry changes communication, most readily evident in technical limitations. Camera positioning gives the impression that, while looking directly at the screen, we are not quite looking the patient in the eye. The two-dimensional image lacks depth and impairs facial observation. As emotions are closely linked to expressions - and given the role of the face in human communication in general-this impairment is especially compromising to residents who are still learning to identify subtle affective cues and improve their own communication [2].

Trust is more difficult to develop, amid the usual difficulties experienced by the constantly rotating resident. Delays in data transmission interrupt speech and expressions, damaging the flow of the interview. Some of my patients with paranoid ideations have feared for their privacy because the session is conducted over the Internet. The omission of peripheral body language (e.g., being unable to empathically interrupt the talkative patient with one's hands) has been cited as a threat to the therapeutic relationship and may impair our ability to practice rapport building [3]. With the patient with neurocognitive or sensory problems, technical difficulties only aggravate an already challenging situation.

The interaction is also affected by our separation in location. Although transportation can be stressful, it provides a

J. Alexander Scott

jamsco@med.umich.edu

1 University of Michigan, Ann Arbor, MI, USA transition from which we step out of our daily affairs to participate in treatment. The loss of this transition can devalue the session, such as when patients present while driving, or in the company of another (who would not otherwise accompany them), or during a break at the workplace, or while babysitting children. These scenarios (which I experienced many times over) create obvious demands on attention and impair the patient's ability to reflect and tolerate distress.

In the virtual setting, the resident psychiatrist must manage treatment boundaries previously taken for granted. Confidentiality and privacy may be threatened if the patient is not alone. Patient engagement during those morning appointments in which they awoke only moments ago is compromised (as anyone who has "pre-rounded" before dawn in the hospital can attest). We residents risk perpetuating these boundary violations as well, especially if practicing from a home office. New problems arise with virtual visits enabling avoidance of the public in patients suffering from anxiety or psychotic disorders. In gently confronting these patients to encourage progress, I have required frequent guidance from my supervisors.

Experience in clinical skill and medical decision-making is affected in the virtual setting. Vital signs, urine drug tests, electrocardiograms, and metabolic profiles have all proved difficult for me to obtain in the patient's absence. Many of my patients have been unable to obtain serum levels of medication with regular frequency. I have lost experience in interpreting these measures and discussing them with patients. Ad hoc, poor lighting conditions obscure expressions and details of appearance. Our relative inability to view the patient's entire body costs us valuable experience assessing tics, tremors, and gait. Neuropsychiatric examinations such as those assessing for catatonia or extrapyramidal effects of medications are difficult to perform.

It can be challenging to ensure patient safety in the virtual interaction. It is easier for a resident to arrange a safe disposition with physical assistance from other parties (security personnel, nurses, and patient family members). Uncertainty arises about when to contact law enforcement (or where to direct them, in the case of one patient who 
presented from their car but was unaware of their specific location). Patients can abruptly end virtual visits but could not act so impulsively in an office. Without this co-location of staff and patients, I have diminished training in managing outpatient crises.

In conducting virtual visits, my physical wellness has also suffered, having sat alone at the office for extended periods of time. Practicing telepsychiatry approximates a workday spent almost entirely on the computer, as seeing patients, documenting clinical encounters, and administrative work all become electronic tasks. The discussion around "Zoom fatigue" is ongoing, but the hypothesized negative effects on attention and communication threaten our ability to care for patients optimally, and to learn from didactic sessions and supervision [4].

Virtual meetings may not be as fulfilling as in-person meetings (perhaps due to problems in communication as discussed above). Loneliness, which has been linked to resident burnout and has increased throughout the pandemic, has been associated with physical and mental illness [5]. Many days I walked into an empty building in the morning, saw patients, and returned to the parking lot in the evening without having seen another employee all day. It is not yet clear how resident loneliness has fluctuated during the pandemic (e.g., perhaps more activity at hospitals mitigates the internal medicine resident's risk), but I suspect that in psychiatry, it will have increased.

Telepsychiatry has taken a great leap forward as the COVID-19 pandemic necessitated its widespread use, though the degree to which residency training programs will incorporate it remains to be seen. There are certainly benefits to virtual visits, and administrators have worked hard to give residents the best learning experience amid the chaos.
As telepsychiatry is adopted nationally, it will become prudent for us to gain more experience in a hybrid model of virtual and in-person visits. Yet, the virtual setting and its effects on training must be examined more closely, as clinical successes do not necessarily ensure educational ones.

\section{Declarations}

Disclosures The author states that there is no conflict of interest.

\section{References}

1. Totura C, Fields SA, Karver MS. The role of the therapeutic relationship in psychopharmacological treatment outcomes: a metaanalytic review. Psychiatr Serv. 2018;69(1):41-7.

2. Russell JA, Bachorowski JA, Fernandez-Dols JM. Facial and vocal expressions of emotion. Annu Rev Psychol. 2003;54:329-49.

3. Ghosh A, Sharma K, Choudhury S. COVID-19 and physicianpatient relationship: potential effects of 'masking', 'distancing' and 'others.' Fam Pract. 2021;38(2):193-4.

4. Lee J. A neuropsychological exploration of Zoom fatigue. Psychiatric Times. Available from: https://www.psychiatrictimes.com/ view/psychological-exploration-zoom-fatigue [Accessed July 27th, 2021].

5. Shapiro J, Zhang B, Warm EJ. Residency as a social network: burnout, loneliness, and social network centrality. J Grad Med Educ. 2015;7(4):617-23.

Publisher's Note Springer Nature remains neutral with regard to jurisdictional claims in published maps and institutional affiliations. 